\title{
Cannabinoids Ameliorate Impairments Induced by Chronic Stress to Synaptic Plasticity and Short-Term Memory
}

\author{
Hila Abush' and Irit Akirav*,' \\ 'Department of Psychology, University of Haifa, Haifa, Israel
}

\begin{abstract}
Repeated stress is one of the environmental factors that precipitates and exacerbates mental illnesses like depression and anxiety as well as cognitive impairments. We have previously shown that cannabinoids can prevent the effects of acute stress on learning and memory. Here we aimed to find whether chronic cannabinoid treatment would alleviate the long-term effects of exposure to chronic restraint stress on memory and plasticity as well as on behavioral and neuroendocrine measures of anxiety and depression. Late adolescent rats were exposed to chronic restraint stress for 2 weeks followed each day by systemic treatment with vehicle or with the CBI/2 receptor agonist WIN55,2 $12-2$ (1.2 mg/kg). Thirty days after the last exposure to stress, rats demonstrated impaired long-term potentiation (LTP) in the ventral subiculum-nucleus accumbens (NAc) pathway, impaired performance in the prefrontal cortex (PFC)-dependent objectrecognition task and the hippocampal-dependent spatial version of this task, increased anxiety levels, and significantly reduced expression of glucocorticoid receptors (GRs) in the amygdala, hippocampus, PFC, and NAc. Chronic WIN55,2I2-2 administration prevented the stress-induced impairment in LTP levels and in the spatial task, with no effect on stress-induced alterations in unconditioned anxiety levels or GR levels. The CBI antagonist AM25I $(0.3 \mathrm{mg} / \mathrm{kg})$ prevented the ameliorating effects of WIN55,2I2-2 on LTP and short-term memory. Hence, the beneficial effects of WIN55,2I 2-2 on memory and plasticity are mediated by CBI receptors and are not mediated by alterations in GR levels in the brain areas tested. Our findings suggest that cannabinoid receptor activation could represent a novel approach to the treatment of cognitive deficits that accompany a variety of stress-related neuropsychiatric disorders.

Neuropsychopharmacology (2013) 38, I52 I-1534; doi: I0.1038/npp.20 I3.5 I; published online I3 March 2013
\end{abstract}

Keywords: chronic stress; cannabinoids; LTP; short-term memory; glucocorticoid receptors; WIN55,2। 2-2

\section{INTRODUCTION}

Repeated life stress precipitates and exacerbates mental illnesses, including depression and anxiety (Dinan, 2005). Homotypic stressors that occur on a daily basis are associated with increased depressive symptoms (Caspi et al, 2003; Hammen et al, 2004; Hammen, 2005; Keller et al, 2007; Robertson Blackmore et al, 2007; Southwick et al, 2005). Repeated restraint stress is an animal model that recapitulates anxious and depressive symptoms, and associated physiological changes, and has allowed for experimental investigations into the contributions of stress to mental illness and the mechanisms underlying stress-adaptation (Patel and Hillard, 2008).

When examining the short-term effects of chronic stress (between $24 \mathrm{~h}$ and 14 days after exposure to stress), repeated restraint stress has been shown to induce depressive and anxious phenotypes (Kim and Han, 2006; Mitra et al, 2005; Vyas and Chattarji, 2004) and cognitive impairments (Lupien et al, 2007; Radecki et al, 2005; Wright

*Correspondence: Dr I Akirav, Department of Psychology, University of Haifa, Mt Carmel, Haifa 31905, Israel, Tel: +972 48288268 , Fax: + 97248263157 , E-mail: irit.akirav@gmail.com

Received 10 October 2012; revised 27 January 2013; accepted 14 February 2013; accepted article preview online 20 February 2013 and Conrad, 2008), symptoms often experienced in depressive illness (Irwin and Miller, 2007; Mathews and MacLeod, 2005; Schneiderman et al, 2005). Chronic stress impairs spatial as well as working memory, suggesting negative effects on prefrontal cortex (PFC) as well as on hippocampal functioning (Conrad et al, 1996; Mizoguchi et al, 2000; Park et al, 2001).

The endocannabinoid system has recently emerged as a promising therapeutic target for the treatment of stress-related emotional disorders (Abush and Akirav, 2010; Ganon-Elazar and Akirav, 2009; 2012; Gorzalka et al, 2008; Hill et al, 2010; Patel et al, 2005; Ramot and Akirav, 2012; Segev et al, 2012). Cannabinoid receptor activation in the basolateral amygdala (BLA) using the CB1/2 receptor agonist WIN55,212-2 prevented the effects of acute stress on emotional learning (Ganon-Elazar and Akirav, 2009; 2012; Ramot and Akirav, 2012). WIN55,212-2 also inhibited stress-induced elevation in corticosterone levels and stressinduced alterations in hypothalamic-pituitary-adrenal (HPA) negative feedback (Ganon-Elazar and Akirav, 2009; 2012). Chronic cannabinoids were shown to enhance stresscoping responses and to exert long-term antidepressant effects (Bortolato et al, 2007; Macri and Laviola, 2004).

There are also indications that chronic stress exposure affects endocannabinoid levels. Exposure to daily restraint 
episodes activated 2-AG-mediated signaling in the medial PFC, amygdala, hippocampus, and hypothalamus (Patel et al, 2005; Rademacher et al, 2008). Chronic treatment of rats with corticosterone also resulted in increased 2-AG content in the amygdala (Hill et al, 2005). In the medial PFC and amygdala, restraint stress decreased $\mathrm{N}$-arachidonylethanolamine content regardless of the number of previous exposures (Patel et al, 2005; Rademacher et al, 2008). Repeated immobilization stress exposure increased CB1 mRNA and protein expression in the PFC of WT mice and daily pretreatment with the selective CB1 agonist arachidonyl-2-chloroethylamide prevented this stress-induced upregulation (Zoppi et al, 2011).

Long-term potentiation (LTP) is one of the prime candidates for mediating learning and memory as well as many other forms of experience-dependent plasticity. The successful $v s$ unsuccessful induction of LTP can serve as a 'diagnostic' measure with which to assess the functional state of a brain structure (Diamond et al, 2007). Growing attention has been focused on plasticity in the ventral subiculum (vSub)-nucleus accumbens (NAc) pathway (Abush and Akirav, 2012; O'Donnell and Grace, 1995). The hippocampus is particularly sensitive to stress (Garcia et al, 1997; Maroun and Richter-Levin, 2003) and is highly interconnected with the HPA axis response. In addition to the hippocampus, other brain structures have been implicated in a neural circuitry of stress, including the amygdala, PFC, and NAc. The NAc is involved in mediating stress-related dysfunction (Nestler et al, 2002; Willner et al, 1992; Wood et al, 2004; Zangen et al, 2001) and, with input from the vSub, mediates goal-directed behavior and is important for aspects of cognitive function, such as contextdependent processing (Belujon and Grace, 2008). This pathway is also involved in spatial and object recognition (Léna et al, 2001; Poucet et al, 2004; Sargolini et al, 2003). Specifically, it has been suggested that the hippocampus allows for place recognition and the storage of the paths to get there, and the NAc translates paths in neural space into appropriate locomotor activity that moves the animal towards the goal in real space.

Recently, we found that the impairing effects of chronic WIN55,212-2 on short-term memory in the water maze and the object-recognition tasks as well as LTP in the vSub-NAc pathway were temporary as they lasted only $24 \mathrm{~h}$ or 10 days after withdrawal (Abush and Akirav, 2012). However, chronic WIN55,212-2 significantly impaired hippocampaldependent short-term memory measured in the objectlocation task even 75 days after the last drug injection. These suggest that most cognitive impairments induced by WIN55,212-2 are temporary and probably result from a residue of cannabinoids in the brain or acute withdrawal effects. This also suggests that the effects of chronic WIN55,212-2 on hippocampal-dependent function are task-dependent and specific.

Many disease states feature HPA axis dysregulation in the form of changes in glucocorticoid receptors (GRs) levels, basal corticosterone secretion, or feedback regulation. Both GR and CB1 receptors are located within the stress circuitry in the brain (ie, amygdala, hippocampus, and PFC) (Ahima and Harlan, 1990; Herkenham et al, 1990; Katona et al, 2001; van Eekelen et al, 1987). There is a critical and complex role of the endocannabinoid system in the regulation of HPA axis activity and glucocorticoid-mediated negative feedback (for review, see Hill and Tasker, 2012). Under conditions of chronic stress the endocannabinoid system contributes to multiple forms of HPA axis regulation that involve endocannabinoid signaling in the amygdala and PFC (Hill et al, 2010; Patel et al, 2004; Patel et al, 2005). Structures within the forebrain limbic system (ie, hippocampus, amygdala, and $\mathrm{PFC}$ ), have an integral role in regulating this system (Herman et al, 2005). Hence, alterations in GRs have a significant influence on HPA axis activity, particularly by modulating the strength of negative feedback and therefore the regulation of glucocorticoid levels (Yehuda et al, 2012). Previously, it has been shown that chronic stress decreased hippocampal GRs (Sapolsky et al, 1984).

In this study, we aimed to examine whether chronic treatment with cannabinoids in late adolescent rats could prevent the long-term effects of chronic exposure to repeated restraint-stress on cognitive function, including synaptic plasticity in the vSub-NAc pathway and short-term memory. Our previous results suggest that the preventing effects of WIN55,212-2 on stress-induced alterations in emotional memory are mediated via the HPA axis (GanonElazar and Akirav, 2009; 2012) and specifically GRs in the amygdala and hippocampus (Ganon-Elazar and Akirav, 2013). Here we aimed to examine the effects of chronic stress on GR levels in the forebrain limbic system and to test whether WIN55,212-2 would prevent these stress-induced effects. This would allow exploring the possibility that the preventing effects of WIN55,212-2 on plasticity and shortterm memory are associated with alterations in GRs in limbic areas. In order to examine the long-term effects of stress and cannabinoids, rats were tested 30 days after stress and drug treatment ended. Hence, we examined the longterm effects of cannabinoids that persist after drug use stops and after withdrawal.

\section{MATERIALS AND METHODS}

\section{Subjects}

Male Sprague-Dawley rats (45-days old, $\sim 200 \mathrm{~g}$ ), caged together at $22 \pm 2{ }^{\circ} \mathrm{C}$ under 12-h light/dark cycles (lights turned on at 1900 hours). Rats had access to water and laboratory rodent chow ad libitum, except during the sucrose intake test. The experiments were approved by the University of Haifa Ethics and Animal Care Committee, and adequate measures were taken to minimize pain or discomfort.

\section{Chronic Stress}

During post-natal days $45-60$, the rats were restrained for $1 \mathrm{~h}$ every day (approximately at 1200 hours each day), for 14 days. Rats were placed in clear acrylic cylindrical tubes (length: $13 \mathrm{~cm}$; diameter: $6 \mathrm{~cm}$ ), allowing only for breathing and minimal movements of the limbs.

\section{Drug Treatment}

Rats received 14 injections of vehicle or WIN55,212-2, one per day, $1 \mathrm{~h}$ after restraint stress ended. WIN55,212-2 (Tocris, USA) was initially dissolved in 1\% Tween 80 and in 
$1 \%$ DMSO and further diluted with saline $(0.9 \% \mathrm{NaCl})$. The vehicle solution contained 1\% DMSO, $1 \%$ Tween 80 , and $98 \%$ saline. WIN55,212-2 was administered intraperitoneally at a dose of $1.2 \mathrm{mg} / \mathrm{kg}, 0.3 \mathrm{ml}$. WIN55,212-2 dose was based on previous reports (Abush and Akirav, 2012; Schneider and Koch, 2007; Schneider et al, 2008). The CB1 receptor antagonist AM251 (0.3 mg/kg; Tocris Bioscience) was co-administered with WIN55,212-2.

\section{Object-Location Memory Task}

This task measures an animal's ability to detect that an object has moved to a new location (described in Abush and Akirav, 2012). The objects were two small identical ceramic dolls located in a squared black open field $(50 \times 50 \times 50 \mathrm{~cm})$ under dim light. The rats were habituated to the open field by allowing them to explore it for $10 \mathrm{~min}$ every day for 4 days (without objects).

In the sample phase, each rat was exposed to the objects for $5 \mathrm{~min}$. After $30 \mathrm{~min}$ (test phase), one object was moved to a new location and the time spent exploring the objects at the old and new locations were recorded for $5 \mathrm{~min}$.

A discrimination index (DI) calculated for each animal was expressed as $\mathrm{TN} /(\mathrm{TN}+\mathrm{TF})$ where $\mathrm{TF}=$ time exploring familiar location; and $\mathrm{TN}=$ time exploring novel location. Intact spatial recognition memory in the test phase was reflected in a discrimination score $>50 \%$, which implies greater exploration of the object in the novel location.

\section{Object-Recognition Memory Test}

This task measures the ability to discriminate the familiarity of previously encountered objects. All parameters were identical to the object-location task described above, except for the test phase in which the rat was presented with one of the objects from the sample trial and with a novel object for $5 \mathrm{~min}$.

\section{Electrophysiology}

The procedure is as described in Abush and Akirav (2012). Rats were anesthetized (40\% urethane, 5\% chloral hydrate in saline; $4 \mathrm{ml} / 1 \mathrm{~kg}$, i.p.) and placed in a stereotaxic frame. A recording microelectrode (glass, tip diameter of $2-5 \mu \mathrm{m}$, filled with $2 \mathrm{M} \mathrm{NaCl}$, resistance of 1-4 M) was inserted through small burr holes into the NAc shell (anteroposterior, $\pm 1.6 \mathrm{~mm}$; lateral, $\pm 1.0 \mathrm{~mm}$; ventral, $-5.5 \mathrm{~mm}$ ). A bipolar $125-\mu \mathrm{m}$-stimulating electrode was positioned in the vSub (anteroposterior, $-6.5 \mathrm{~mm}$; lateral, $\pm 5.0 \mathrm{~mm}$; ventral, $-6.0 \mathrm{~mm})$. The evoked responses were digitized $(10 \mathrm{kHz})$ and analyzed using the Cambridge Electronic Design (CED, Cambridge, UK) $1401+$ and its Spike2 software. Offline measurements were made of the amplitude and slope of the excitatory postsynaptic potentials (EPSPs) using averages of five successive responses to a given stimulation intensity applied at $0.1 \mathrm{~Hz}$. Test stimuli (monopolar pulses, $100 \mu \mathrm{s}$ duration) were delivered at $0.1 \mathrm{~Hz}$. After positioning the electrodes, the rats were left for $1 \mathrm{~h}$ before commencing the experiment.

LTP was induced by theta-like high-frequency stimulation (HFS) (three sets of 10 trains; each train consisting of 10 pulses at $200 \mathrm{~Hz}$; inter-train interval, $200 \mathrm{~ms}$; inter-set interval, $1 \mathrm{~min}$ ) to the vSub. LTP was measured as an increase in amplitude and slope of EPSPs. Potentiation was measured as a percentage change from the average of the 30-min baseline before HFS.

\section{Open Field}

The floor of the squared black open field $(50 \times 50 \times 50 \mathrm{~cm})$ was divided by $1-\mathrm{cm}$-wide white lines into 25 squares measuring $10 \times 10 \mathrm{~cm}$ each. Recordings were made over a period of $5 \mathrm{~min}$ and the time the rat spent in the central and peripheral squares and the total distance covered were measured.

\section{Forced Swim}

Rat was forced to swim for 15 min inside a vertical clear acrylic cylinder (height: $50 \mathrm{~cm}$; diameter: $20 \mathrm{~cm}$ ) containing $34 \mathrm{~cm}$ of water maintained at $\sim 22^{\circ} \mathrm{C}$. After $24 \mathrm{~h}$ in the home cage, the rat was put back in the cylinder and forced to swim for $5 \mathrm{~min}$. The time spent climbing, swimming, or immobile was recorded and analyzed. Immobility was defined as the lack of motion, except for movements necessary to keep the animal's head above water.

\section{Sucrose Intake}

Water bottles were removed before the dark part of the cycle and replaced with bottles containing a 1\% sucrose solution. Sucrose consumption was measured during the 12 dark hours of the cycle and was then normalized according to every rat's specific weight. Four measurements of sucrose consumption were taken: before stress and injections, $24 \mathrm{~h}, 10$ days, and 30 days after the last exposure to stress and injections.

\section{Biochemical Methods}

Thirty days after the last stress and/or drug injections, the rats were killed and their brains were immediately frozen at $-80{ }^{\circ} \mathrm{C}$ for western blot analysis. No anesthesia was used before decapitation. Brain tissues of the medial PFC, NAc, BLA, CA1, and vSub were then collected and homogenized in lysis buffer ( $10 \mathrm{mM}$ Hepes, $2 \mathrm{mM}$ EDTA, $2 \mathrm{mM}$ EGTA, $0.5 \mathrm{mM}$ DTT, $10 \mathrm{ml} / \mathrm{ml}$ leupeptin, $10 \mathrm{ml} / \mathrm{ml}$ aprotinin). Protein levels were determined by the bicinchoninic acid (BCA) Protein Assay Kit (Pierce) according to the manufacturer's protocol. The samples were then diluted in SDS sample buffer, boiled $\left(100{ }^{\circ} \mathrm{C}\right)$ for $5 \mathrm{~min}$, and stored at $-80^{\circ} \mathrm{C}$. Aliquots were subjected to SDS-PAGE (7.5\% polyacrylamide) and immunoblot analysis. Blots were incubated with the GR antibody overnight at $4{ }^{\circ} \mathrm{C}(1: 200$, Pierce Antibodies) followed by washing and $1 \mathrm{~h}$ incubation with an HRP-linked secondary antibody at room temperature (donkey anti-goat IgG and goat anti-rabbit IgG; Jackson ImmunoResearch Laboratories, 1:10000). Blots were visualized by enhanced chemiluminescence with ECL (Biological Industries) and quantified with an XRS chargecoupled device camera (Bio-Rad Laboratories) and Quantity One software. All protein samples were standardized with $\beta$-actin (1:5000, polyclonal goat antibody; Santa Cruz Biotechnology, USA). 


\section{Statistical Analysis}

The results are expressed as means \pm SEM. For statistical analysis, one-way ANOVA, two-way ANOVA, mixed design three-way ANOVA, and one-sample $t$-test were used as indicated. All post-hoc comparisons were made using the least significant difference (LSD) multiple-comparison test.

\section{Experimental design}

\begin{tabular}{lccc}
\hline & $\begin{array}{c}\text { Chronic stress and/or } \\
\text { WIN administration }\end{array}$ & 30 days rest & Testing \\
\hline Experiment days & $\mid-14$ & $15-44$ & 45 \\
(Post-natal days) & $(45-60)$ & $(61-90)$ & $(91)$ \\
\hline
\end{tabular}

During post-natal days $45-60$, the rats were restrained for $1 \mathrm{~h}$ every day, for 14 days and during this period received 14 injections of vehicle or WIN55,212-2 (or AM251 and WIN55,212-2), one per day, $1 \mathrm{~h}$ after restraint stress ended. Each test (except for Exp3) was performed on different sets of rats to prevent carryover effects due to multiple tests. Thirty days after the last stress exposure and injection, rats were taken to the following experiments:

Exp1: LTP in the vSub-NAc pathway (Figure 1).

Exp2: Object-location task and object-recognition task (Figure 2).

Exp3: Locomotion and anxiety in the open field (Figure 3) and forced-swim test (Figure 4a). In the forced-swim test, testing was performed $24 \mathrm{~h}$ (Figure $3 \mathrm{~b}$ ) or 10 days (Figure 3c) after the last stress exposure and injection. Figure 5 shows sucrose consumption and weight gain at the different time points ( $24 \mathrm{~h}, 10$ days, and 30 days).

Exp4: Western blots to measure GR levels in the NAc, BLA, PFC, CA1, and vSub (Figure 6).

\section{RESULTS}

\section{The Effects of Chronic Exposure to Restraint Stress and} WIN55,212-2 on Synaptic Plasticity

Rats were exposed to chronic restraint stress followed by vehicle (Stress-Vehicle) or WIN55,212-2 (Stress-WIN) injections, or merely injected with vehicle (No StressVehicle) or WIN55,212-2 (No Stress-WIN) with no stress exposure.

For LTP, the data were analyzed using mixed design three-way ANOVA with Drug (vehicle/WIN) and Stress (No Stress/Stress) as between-subject factors, Time as within-subject factor, and EPSP amplitude and slope levels as dependent variables (Figure 1).

The amplitude was measured by peak-to-peak values based on previous findings on LTP in the vSub-NAc pathway (Dong et al, 2007; Abush and Akirav, 2012). However, it is the EPSP slope that is considered to reflect LTP (ie, the strengthening of existing synaptic contacts) while the amplitude may also reflect the activity of the cells.

Post-HFS analysis $(($ Drug $\times$ Stress $\times$ Time $(2 \times 2 \times 12))$ of the amplitude and slope indicated a significant Drug $\times$ Stress interaction (amplitude: $\mathrm{F}(1,28)=8.62, p<0.01$; slope: $\mathrm{F}(1,28)=5.27, p<0.05)$. There were no significant effects for Drug, Stress, Time, Time $\times$ Drug, Time $\times$ Stress, or Time $\times$ Drug $\times$ Stress for the amplitude (Figure 1a). For the slope, there was a significant effect for Time $(\mathrm{F}(1,28)=23.49, \quad p<0.001)$, but not for Drug, Stress, Time $\times$ Drug, $\quad$ Time $\times$ Stress, or Time $\times$ Drug $\times$ Stress (Figure 1b).

Post-hoc comparison revealed significantly lower potentiation in the Stress-Vehicle group compared with the No Stress-Vehicle (amplitude: $p<0.01$; slope: $p=0.053$ ) and Stress-WIN groups $(p<0.01)$, suggesting that exposure to WIN55,212-2 resulted in the significant recovery of LTP in the stress group. No significant differences were found between the No Stress-WIN group and the No StressVehicle and Stress-WIN groups, suggesting that WIN55,212-2 by itself had no effect on plasticity.

Furthermore, one-sample $t$-test performed on potentiation levels post HFS in the No Stress-WIN group revealed a significant difference from the $100 \%$ baseline in the amplitude and slope $(p<0.05)$, suggesting significant LTP levels in this group.

Three-Way mixed design ANOVA on EPSP amplitude pre-HFS $($ Drug $\times$ Stress $\times$ Time $(2 \times 2 \times 6))$ did not reveal significant effects for Drug, Stress, Time, or any of the interactions between them $(F(1,28)<1$, NS) for the amplitude, but revealed a significant effect for Time $(\mathrm{F}(1,28)=28.87, p<0.001)$, for the slope.

Figure 1c shows representative traces in the NAc taken before and $1 \mathrm{~h}$ after HFS to the vSub.

As WIN55,212-2 is a CB1/CB2 agonist, we examined whether the preventing effects of WIN55,212-2 on plasticity are mediated by the $\mathrm{CB} 1$ receptor by using a combination of WIN55,212-2 and a CB1 receptor antagonist AM251 $(0.3 \mathrm{mg} / \mathrm{kg})$. Rats were exposed to chronic restraint stress followed by co-administration of AM251 and WIN55,212-2 (Stress-AM + WIN). Mixed design ANOVA (treatment $\times$ time $(4 \times 12))$ post-HFS analysis indicated a significant effect for treatment on the amplitude and slope $(p<0.05)$. Post-hoc comparisons revealed that the Stress-Vehicle group demonstrated significantly lower potentiation compared with the No Stress-Vehicle $(p<0.01)$ and Stress-WIN $(p=0.05)$ groups (Figures $1 \mathrm{~d}$ and e). Also, for the slope, the Stress-AM + WIN group demonstrated marginally significantly lower potentiation compared with the No StressVehicle group $(p=0.055)$.

When the CB1 antagonist was injected without stress exposure, LTP levels were intact (LTP levels (means \pm SEM) $1 \mathrm{~h}$ post HFS: amplitude: $120.8 \pm 4.9$; slope: $119.9 \pm 2.0$ ) (data not shown)). Taken together, the results suggest that AM251 by itself had no effect on plasticity, but AM251 attenuated (amplitude) or blocked (slope) the ameliorating effects of WIN55,212-2 on plasticity measured post stress.

\section{The Effects of Chronic Exposure to Restraint Stress and WIN55,212-2 on Short-Term Memory}

Next, we examined the effects of chronic exposure to restraint stress and WIN55,212-2 on short-term memory. The data were analyzed using two-way ANOVA with Drug and Stress as between-subject factors $(2 \times 2)$ and DI as the dependent variable.

For the object-location task (Figure 2a), a significant Drug $\times$ Stress interaction was found $(F(1,28)=5.13$, 
a

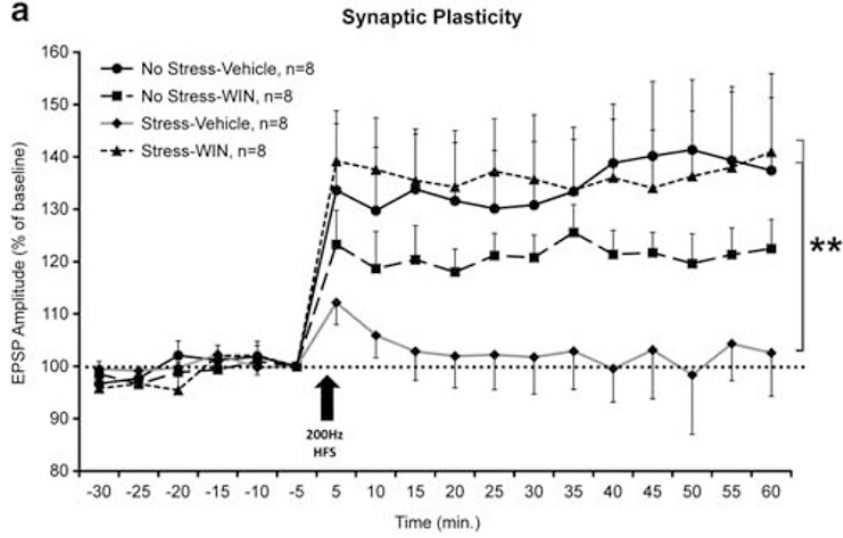

c
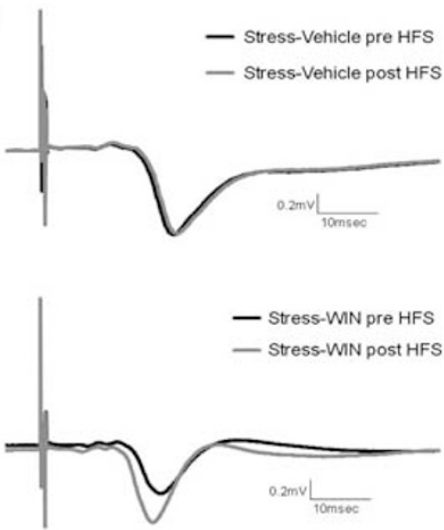

b

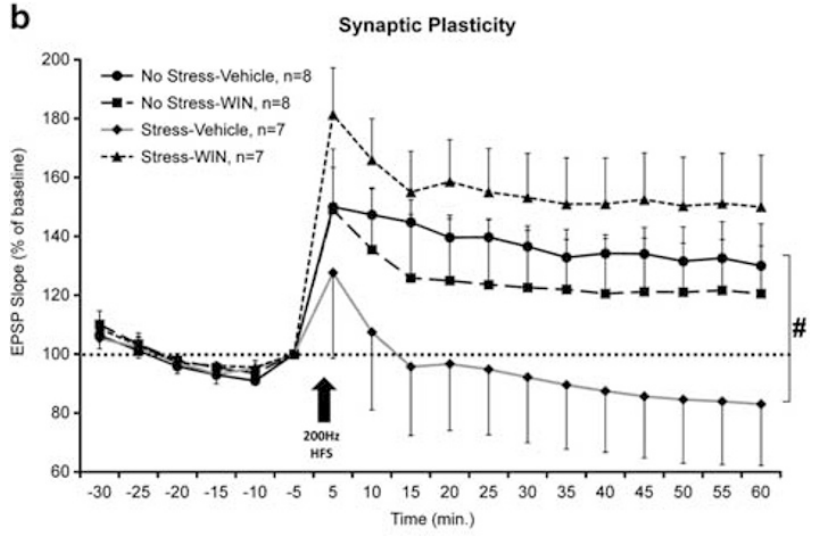

d

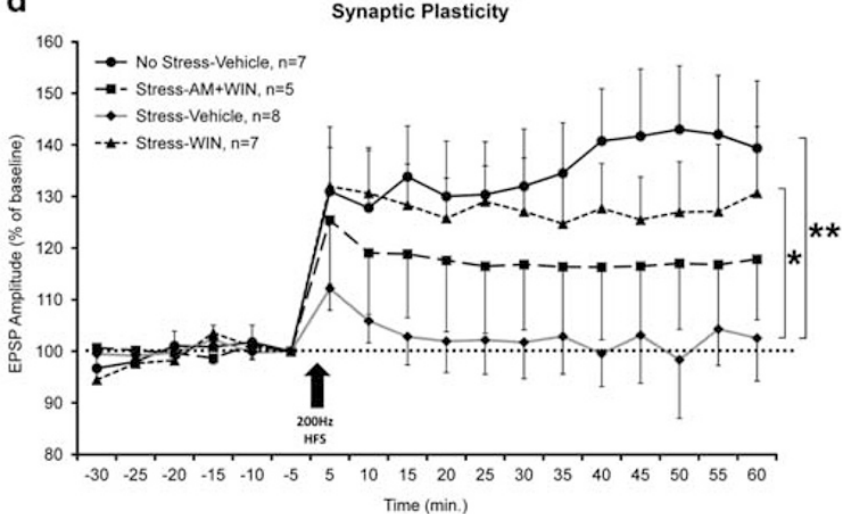

e

Synaptic Plasticity

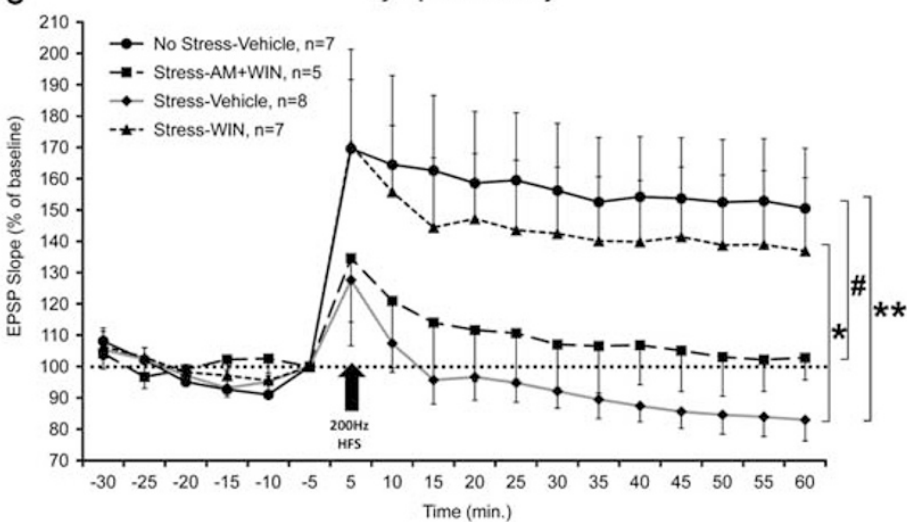

Figure I The effects of chronic exposure to restraint stress and WIN55,2I2-2 on synaptic plasticity in the ventral subiculum-nucleus accumbens pathway. (a) The Stress-Vehicle group demonstrates reduced amplitude levels compared with the Stress-WIN and No Stress-Vehicle groups (** $<0.0 \mathrm{I}$ ). (b) The Stress-Vehicle group demonstrates reduced slope levels compared with the Stress-WIN and No Stress-Vehicle groups (\#p $=0.053$, $* * * 0.01$ ). (c) Representative traces in the NAc taken before (black) and I hr after (gray) HFS to the vSub (calibration: $0.2 \mathrm{mV}$, I 0 ms). Up: the Stress-Vehicle group; Down: the Stress-WIN group. (d) The Stress-Vehicle group demonstrates reduced amplitude levels compared with the Stress-WIN and No Stress-Vehicle groups $(* p<0.05$; *** $<0.01$ ). (e) The Stress-Vehicle group demonstrates reduced slope levels compared with the No Stress-Vehicle and Stress-WIN groups, and the Stress-AM+WIN group demonstrates reduced slope levels compared with the No Stress-Vehicle group (\#p $=0.055$; $* p<0.05$; *** $p<0.01)$

$p<0.05)$. There was no significant main effect for Drug or Stress. Post-hoc comparisons revealed that the StressVehicle group demonstrated less exploration of the novel location than the No Stress-Vehicle group $(p<0.01)$.

One-sample $t$-test performed on each of the groups revealed a significant difference from the $50 \%$ DI in the No Stress-Vehicle $(t(7)=3.66, p<0.01)$ and the Stress-WIN $(t(7)=2.34, p=0.05)$ groups, suggesting intact performance in the task. No such difference was found in the StressVehicle and the No Stress-WIN groups.

There was no significant difference in the sample phase (day 1) between any of the groups in DI (data not shown).

We also examined whether the preventing effects of WIN55,212-2 on memory are mediated by the CB1 receptor by using a combination of WIN55,212-2 and AM251. Hence, rats were exposed to chronic restraint stress followed by 

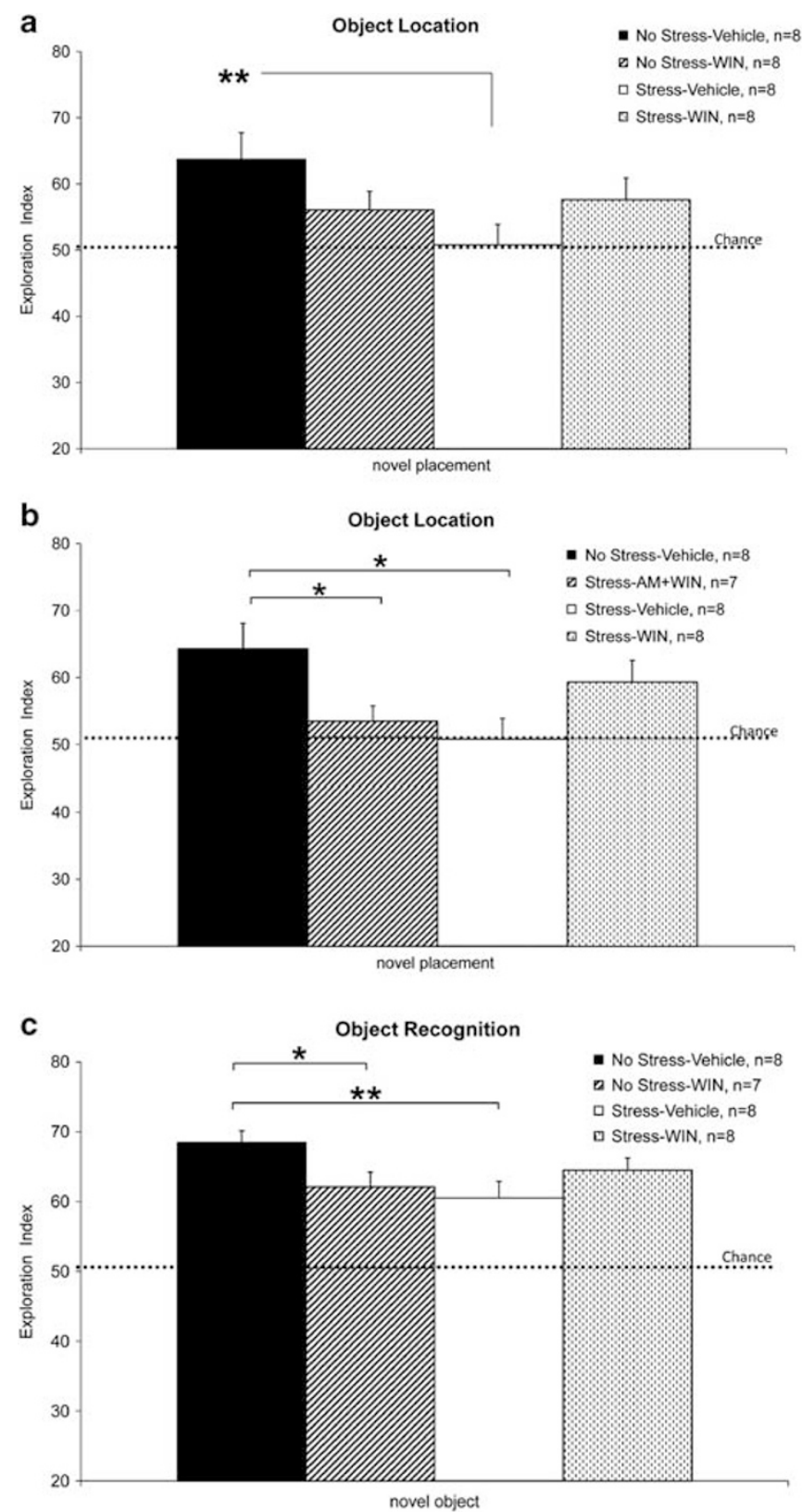

Figure 2 The effects of chronic exposure to restraint stress and WIN55,2 I 2-2 on short-term memory. (a) In the object-location task, rats in the Stress-Vehicle group spend significantly less time exploring the new location compared with rats in the No Stress-Vehicle group. $(* * 2<0.01)$. (b) In the object-location task, the Stress-Vehicle and Stress-AM+WIN groups spend significantly less time exploring the new object compared with rats in the Stress-WIN and No Stress-Vehicle groups $(* p<0.05)$. (c) In the object-recognition task, rats in the No Stress-Vehicle group spend significantly more time exploring the new object compared with rats in the Stress-Vehicle and No stress-WIN groups (* $p<0.05$; $* * p<0.0$ I).

co-administration of AM251 and WIN55,212-2 (Stress$\mathrm{AM}+\mathrm{WIN})$. One-way ANOVA revealed a significant effect for treatment $(\mathrm{F}(3,27)=3.06, p<0.05)$. Post-hoc comparisons revealed that the No Stress-Vehicle group demonstrated significantly more exploration of the novel location than the Stress-Vehicle $(p=0.012)$ and Stress-AM + WIN groups $(p<0.05)$ (Figure $2 b)$. These results suggest that AM251 blocked the ameliorating effects of WIN55,212-2 on post-stress short-term spatial memory.
In the object-recognition task (Figure 2c), a significant Drug $\times$ Stress interaction was found $(\mathrm{F}(1,27)=7.47$, $p=0.011)$. There was no significant main effect for Drug or Stress. Post-hoc comparisons revealed that the No StressVehicle group demonstrated more exploration of the novel object than the Stress-Vehicle group $(p<0.01)$, and the No Stress-WIN group $(p<0.05)$.

One-sample t-test performed on each of the groups revealed a significant difference from the $50 \%$ DI in all the groups (No Stress-Vehicle: $t(7)=11.83, p<0.001$; No Stress-WIN: $t(6)=5.76, \quad p=0.001 ; \quad$ Stress-Vehicle: $t(7)=4.82, p<0.01$; Stress-WIN: $t(7)=8.96, p<0.001)$. Overall, the data suggest that the Stress-Vehicle group demonstrated attenuated performance in the task compared with the No Stress-Vehicle group.

There was no significant difference in the sample phase (day 1) between any of the groups in DI (data not shown).

\section{The Effects of Chronic Exposure to Restraint-Stress and WIN55,212-2 on Behavioral Measures of Anxiety, Despair, Hedonia, and Weight Gain}

Rats were tested for locomotion and anxiety levels in the open field, for depressive-like symptoms in the forced-swim test, for hedonia in the sucrose consumption test, and their weight was monitored. Our previous findings suggest that acute WIN55,212-2 administration can prevent the effects of acute or single prolonged stress on emotional memory, startle response, and alterations in the HPA axis (GanonElazar and Akirav, 2009; 2012; Ramot and Akirav, 2012). However, WIN55,212-2 did not prevent the effects of stress on unconditioned anxiety (Ganon-Elazar and Akirav, 2012). Hence, we aimed to find out in this model whether the preventing effects of WIN55,212-2 can be generalized to other stress-induced alterations in behavior (ie, anxious and depressive symptoms).

In the open field, two-way ANOVA revealed a significant effect for Stress $(F(1,28)=11.49, p<0.01)$ in the time spent in the center, with no significant effect for Drug or Drug $\times$ Stress (Figure 3a). Post-hoc comparisons revealed that the No Stress-Vehicle group spent significantly more time in the center of the open field than the Stress-Vehicle $(p<0.05)$ and the Stress-WIN $(p<0.01)$ groups. The No Stress-WIN group spent significantly more time in the center of the open field than the Stress-Vehicle and Stress-WIN groups $(p<0.05)$.

For rearings in the open field, there was a significant effect for Drug $(\mathrm{F}(1,28)=13.9, p=0.001)$, with no significant effect for Stress or Drug $\times$ Stress (Figure $3 \mathrm{~b}$ ). Post-hoc comparisons revealed that the No Stress-Vehicle group demonstrated less rearing than the No Stress-WIN and Stress-WIN groups $(p<0.01)$ and that the Stress-Vehicle group demonstrated less rearing than the No Stress-WIN and Stress-WIN groups $(p<0.05)$.

For distance covered, there was a significant Drug $\times$ Stress interaction $(\mathrm{F}(1,28)=5.39, p<0.05)$, with no significant effect for Drug or Stress (Figure 3c). Post-hoc comparisons revealed that the No Stress-WIN group covered significantly more distance in the open field than the No Stress-Vehicle group $(p<0.05)$.

In the forced-swim test (Figure 4a), two-way ANOVA revealed that, for climbing and immobility, there were no 
a

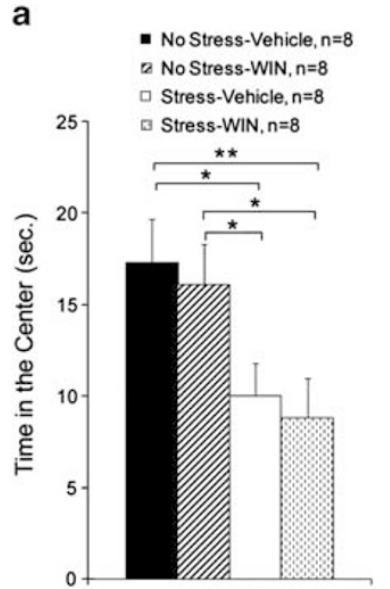

b

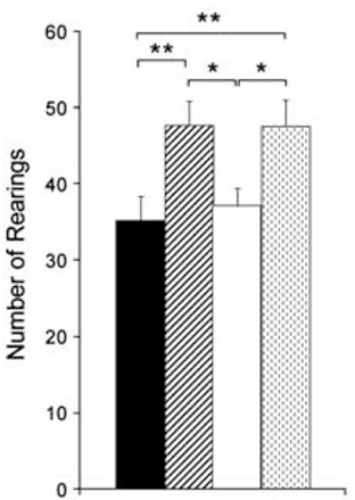

C

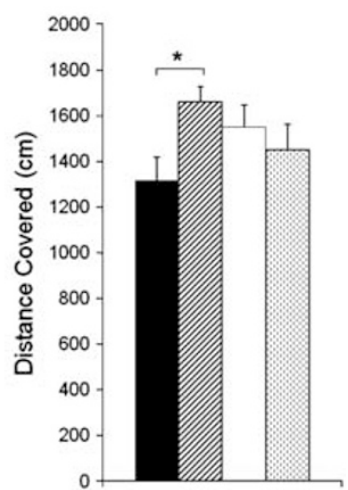

Figure 3 The effects of chronic exposure to restraint-stress and WIN55,212-2 on performance in the open field. (a) Rats in the Stress groups spend significantly less time in the center of the open field than rats in the No Stress groups $(* p<0.05 ; * * *<0.0 I$ ). (b) Rats in the WIN groups demonstrate significantly more rearing than rats in the Vehicle groups ( $p<<0.05$; $* * p<0.0$ I). (c) Rats in the No Stress-WIN group cover more distance in the open field than rats in the No Stress-Vehicle group $(* p<0.05)$.

significant effects for Drug, Stress, or Drug $\times$ Stress $(\mathrm{F}(1,28)<1$, NS). For swimming, there was a significant main effect for Drug $(\mathrm{F}(1,28)=6.86, p=0.014)$. Post-hoc comparisons revealed that rats in the Stress-WIN group spent significantly less time swimming than rats in the Stress-Vehicle and No Stress-Vehicle groups $(p<0.05)$, as measured 30 days after stress exposure ended. There was no significant main effect for Stress $(F(1,28)<1$, NS) or for Drug $\times$ Stress $(\mathrm{F}(1,28)<1, \mathrm{NS})$.

Most studies that measure short-term effects of chronic stress exposure find anxiety- and depression-like behaviors (Kim and Han, 2006). It could be that here the lack of effect in the forced-swim test resulted from the long time frame from the end of chronic stress to the start of behavioral assessment (ie, 30 days). Hence, we performed another experiment in which the performance of rats in the forcedswim test was measured $24 \mathrm{~h}$ and 10 days after cessation of stress/drug exposure.

Two-way ANOVA revealed that $24 \mathrm{~h}$ after stress and WIN55,212-2, there was a significant effect for drug on climbing and immobility ( $p<0.05$; Figure $4 \mathrm{~b})$. Post-hoc comparison revealed that rats in the No Stress-WIN group spent significantly less time climbing than rats in the StressVehicle group $(p<0.01)$ and that rats in the No Stress-WIN group spent significantly more time on immobility than rats in the No Stress-Vehicle and Stress-Vehicle groups $(p<0.01)$. Hence, chronic stress had no effect on performance in the forced-swim test and WIN55,212-2 had a short term effect on motoric behavior as rats injected with WIN55,212-2 (regardless of stress exposure) demonstrated decreased climbing and increased immobility in the $24-\mathrm{h}$ test. Ten days after cessation of chronic stress and drug treatment, there were no significant differences between the groups (Figure $4 \mathrm{c}$ ).

In the sucrose consumption test (Figure 5a), mixed design three-way ANOVA $($ Drug $\times$ Stress $\times$ Time point $(2 \times 2 \times 4))$ at baseline, $24 \mathrm{~h}, 10$ days, and 30 days after exposure to stress revealed no significant main effects for Drug, Stress, or Drug $\times$ Stress $(F(1,28)<1$, NS). There was a significant within-subject effect for Time point $(\mathrm{F}(1,28)=27.11$, $p<0.001$ ), indicating that the rats' sucrose consumption level was significantly higher at the baseline time point. This may result from developmental differences in hedonic sensitivity in late adolescent and adult rats as could be measured by taste reactivity and voluntary consumption (Wilmouth and Spear, 2009).

In weight gain (Figure 5b), mixed design three-way ANOVA $($ Drug $\times$ Stress $\times$ Time point $(2 \times 2 \times 7))$ revealed a significant effect for Stress $(\mathrm{F}(1,28)=24.98, p<0.001)$, Time point $(\mathrm{F}(1,28)=784, p<0.001)$ and the interaction between Stress and Time point $(\mathrm{F}(1,28)=6.77, p<0.05)$. Post-hoc comparison revealed that rats in the No Stress-Vehicle group demonstrated significantly higher weight gain than rats in the Stress-Vehicle and Stress-WIN groups $(p<0.001)$ and that rats in the No Stress-WIN group demonstrated significantly higher weight gain than rats in the StressVehicle and Stress-WIN groups $(p<0.01)$. This indicates that in both vehicle and WIN55,212-2 conditions, rats exposed to chronic stress displayed lower weight gain than rats not exposed to chronic stress.

The Effects of Chronic Exposure to Restraint Stress and WIN55,212-2 on GR Levels in the Nucleus Accumbens, Amygdala, Hippocampus, and Prefrontal Cortex

GR levels were analyzed using two-way ANOVA with Drug and Stress as between-subject factors $(2 \times 2)$. To confirm equal protein loading, the same blots were re-hybridized with antibodies specific for $\beta$-actin. No significant difference in $\beta$-actin levels was observed between the groups, suggesting that $\beta$-actin levels were not affected by the treatment.

In the NAc, there were significant main effects for Drug $(\mathrm{F}(1,24)=5.62, \quad p<0.05) \quad$ and Stress $\quad(\mathrm{F}(1,24)=58.05$, $p<0.001)$, with no significant Drug $\times$ Stress interaction. Post-hoc comparisons of GR levels (Figure 6a) revealed that the stressed groups (Vehicle and WIN) had significantly lower GR levels compared with the No Stress-Vehicle $(p<0.001)$ and No Stress-WIN $(p<0.001)$ groups. Furthermore, the 
a

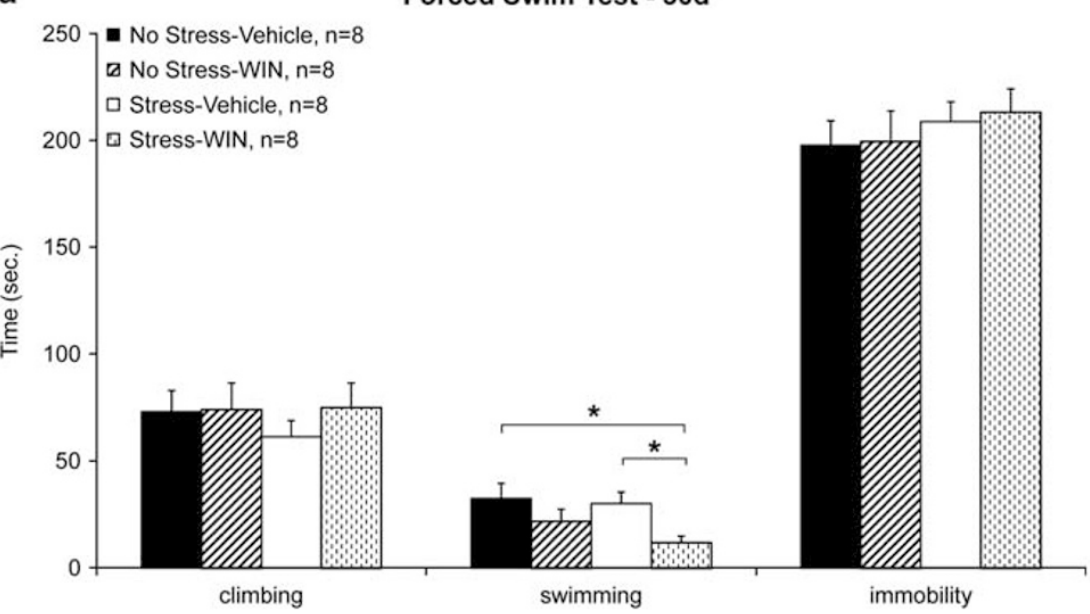

b

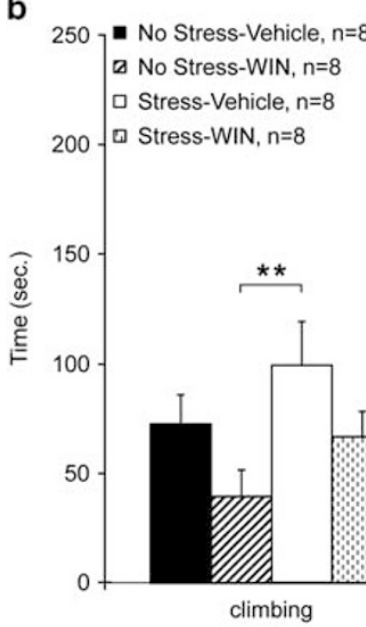

C

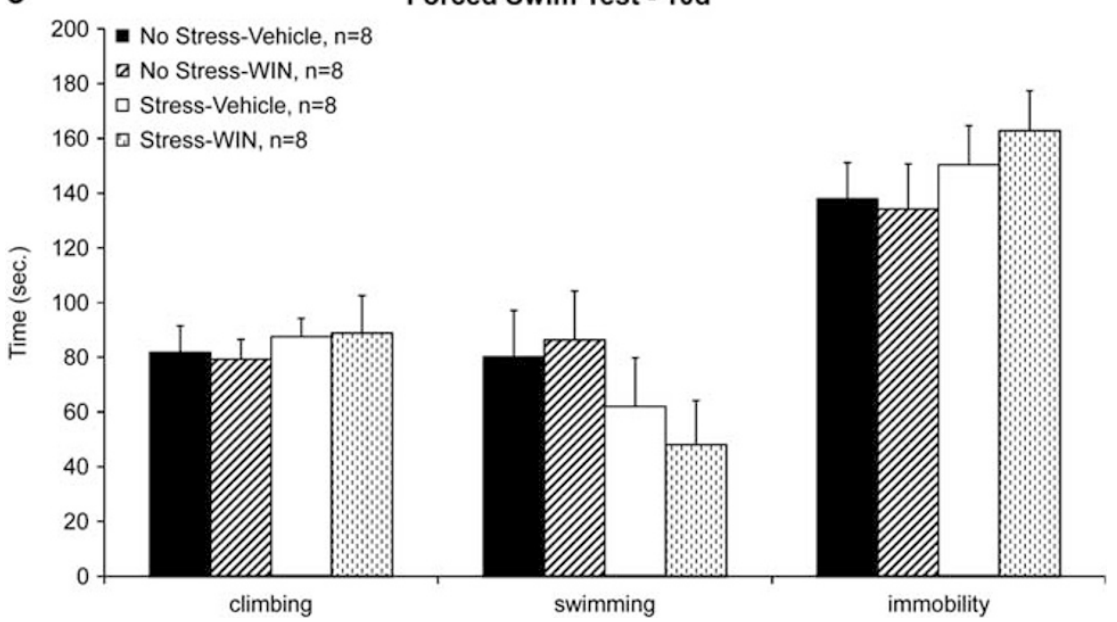

Forced Swim Test - 24h

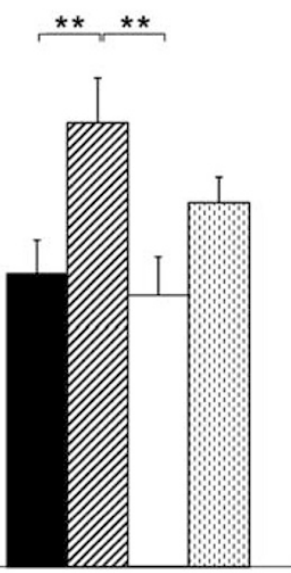

immobility

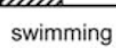

Figure 4 The effects of chronic exposure to restraint-stress and WIN55,212-2 on performance in the forced-swim test. (a) Thirty days after stress and drug injections, rats in the Stress-WIN group spend significantly less time swimming than rats in the Stress-Vehicle and No Stress-Vehicle groups (* $p<0.05$ ). (b) Twenty-four hours after stress and drug injections, rats in the Stress-Vehicle group spend significantly more time climbing than rats in the No Stress-WIN group. Rats in the No Stress-WIN group spend significantly more time immobile than rats in the Vehicle groups (**, $p<0.0 \mathrm{I}$ ). (c) Ten days after stress and drug injections, there are no significant differences between the groups.

No Stress-WIN group had significantly higher GR levels compared with the No stress-Vehicle group $(p<0.01)$.

As chronic administration of WIN55,212-2 increased GR levels in the NAc, we characterized the temporal effect of chronic WIN55,212-2 administration on GR levels. We have previously shown that chronic WIN55,212-2 differentially affected behavior and plasticity when tested $24 \mathrm{~h}, 10$ days, and 30 days after drug treatment ended (Abush and Akirav, 

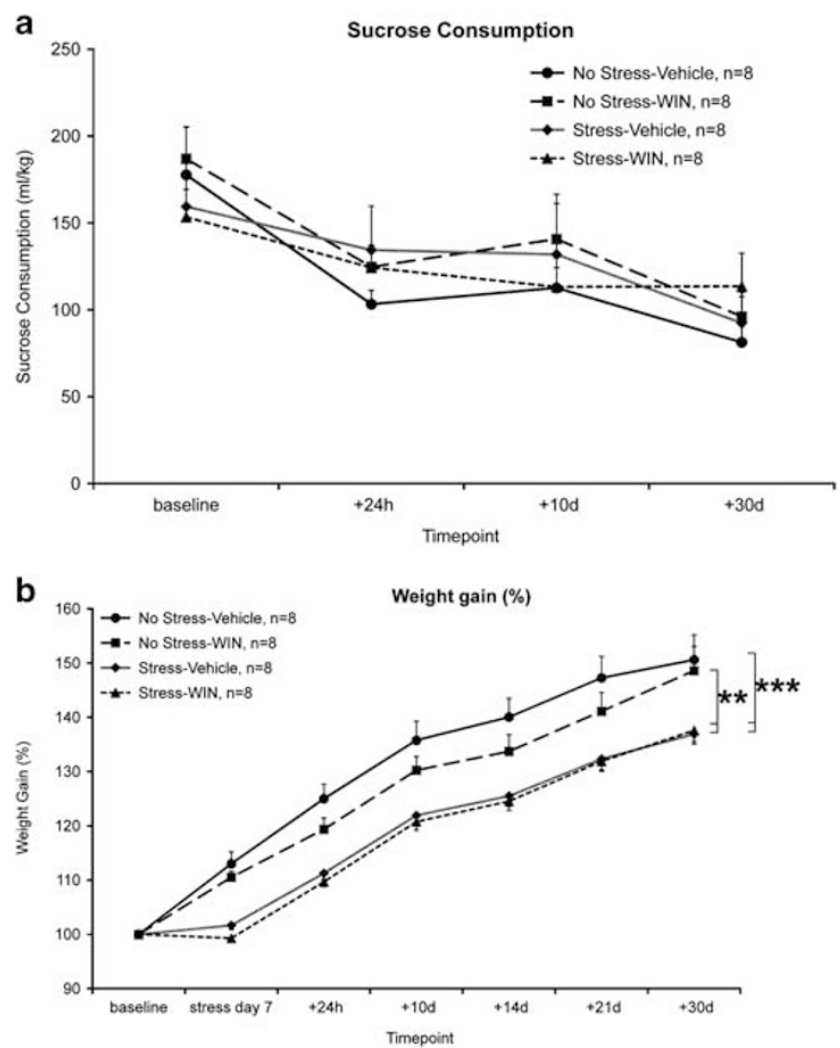

Figure 5 The effects of chronic exposure to restraint-stress and WIN55,212-2 on sucrose consumption and weight gain. (a) There are no significant differences between the groups in the sucrose consumption test. (b) Rats in the stress groups demonstrate lower weight gain rate compared with the non-stressed groups (*** $<0.0$ I; ***** $p<0.00$ I).

2012). Two-way ANOVA of GR levels in the NAc with Drug (vehicle/WIN) and Time point $(24 \mathrm{~h} / 10 \mathrm{~d})$ as betweensubject factors revealed a significant main effect for Drug $(\mathrm{F}(1,21)=7.28, \quad p<0.05)$, with no significant effects for Time point or Drug $\times$ Time point. Post-hoc comparisons $24 \mathrm{~h}$ or 10 days after drug treatment revealed that rats treated with WIN55,212-2 had significantly higher levels of GR than rats treated with vehicle at $24 \mathrm{~h}$ (means \pm SEM: $182.6 \pm 31.8 ; p=0.05)$ and at 10 days $(147.9 \pm 16.3 ; p<0.05$; data not shown).

In the BLA (Figure 6b) and the PFC (Figure 6c), there was a significant main effect for Stress (BLA: $F(1,24)=23.62$, $p<0.001$; PFC: $\mathrm{F}(1,24)=37.67, p<0.001)$, with no significant effects for Drug or Drug $\times$ Stress. Post-hoc comparisons revealed that the Stress-Vehicle group had significantly lower GR levels compared with the No StressVehicle group (BLA: $p<0.05$; PFC: $p<0.001$ ) and compared with the No Stress-WIN group (BLA: $p=0.001$; PFC: $p<0.001)$ and that the Stress-WIN group had significantly lower GR levels compared with the No Stress-WIN group (BLA: $p<0.001$; PFC: $p<0.001$ ) and compared with the No Stress-Vehicle group (BLA: $p<0.01$; PFC: $p<0.001$ ).

When measured $24 \mathrm{~h}$ or 10 days after injections ended, there were no significant effects for Drug, Time point, or Drug $\times$ Time point in the BLA or the PFC (data not shown).

In the CA1 (Figure 6d) and the vSub (Figure 6e), there was a significant main effect for Stress (CA1:
$\mathrm{F}(1,24)=13.57, p=0.001$; vSub: $\mathrm{F}(1,24)=39.11, p<0.001)$, with no significant effects for Drug or Drug $\times$ Stress. Posthoc comparisons revealed that the Stress-Vehicle group had significantly lower GR levels compared with the No StressVehicle group (CA1: $p<0.05$; vSub: $p<0.001$ ) and compared with the No Stress-WIN group (CA1: $p<0.01$; vSub: $p<0.01)$ and that the Stress-WIN group had significantly lower GR levels than the No Stress-WIN group (CA1: $p=0.01$; vSub: $p<0.001)$ and compared with the No StressVehicle group (vSub: $p<0.001$ ).

When measured $24 \mathrm{~h}$ or 10 days after injections ended, there were no significant effects for Drug, Time point, or Drug $\times$ Time point in the CA1 or the vSub (data not shown).

\section{DISCUSSION}

The main finding of this study is that chronic cannabinoid administration can prevent the impairing effects of chronic stress on memory and plasticity, tested 1 month after stress and injections ended. The ameliorating effects of WIN55,212-2 on LTP and short-term memory were prevented by the CB1 antagonist AM251.

Chronic cannabinoid administration did not prevent the stress-induced alterations in GR levels in the NAc, BLA, PFC, and hippocampus, suggesting that the beneficial effects of WIN55,212-2 on memory and plasticity are not mediated by alterations in GR levels in these brain areas.

\section{Synaptic Plasticity and Short-Term Memory}

Chronic restraint-stress resulted in a significant impairment of synaptic plasticity in the vSub-NAc pathway measured 30 days after stress termination. Most studies on the effects of stress on LTP were performed in the hippocampus (Diamond et al, 2005; Foy et al, 2008) and demonstrated impairment. In the vSub-NAc pathway, Dong et al (2007) found that acute stress enabled low-frequency stimulation to induce long-term depression.

Chronic WIN55,212-2 administration by itself did not affect LTP levels tested 30 days after withdrawal, consistent with our previous report that chronic WIN55,212-2 impaired LTP induced $24 \mathrm{~h}$ or 10 days, but not 30 days, after withdrawal (Abush and Akirav, 2012). Importantly, here we show that WIN55,212-2 prevented the stressinduced impairment in LTP, suggesting that chronic cannabinoid treatment might be effective in normalizing cognitive deficits following chronic stress exposure. The CB1 antagonist AM251 blocked the effects of WIN55,212-2 on post-stress EPSP slope, which is considered to better reflect LTP than the EPSP amplitude.

Chronic restraint-stress also impaired hippocampal short-term memory in the spatial location task, when measured 30 days after stress termination, and this effect was shown to be mediated by CB1 receptors. Extensive rodent and human research has shown that the hippocampus is not only crucially involved in memory formation but is also highly sensitive to stress (for review, see Kim and Diamond, 2002). Cognitive deficits resulting from chronic stress and hyperactivation of the HPA axis could be mediated through alterations in hippocampal networks (Aisa et al, 2009; Rothman and Mattson, 2010). 
a No Stress Stress
Vehicle WIN Vehicle WIN

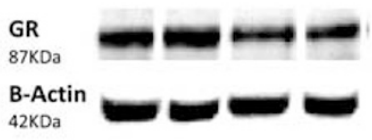

42KDa

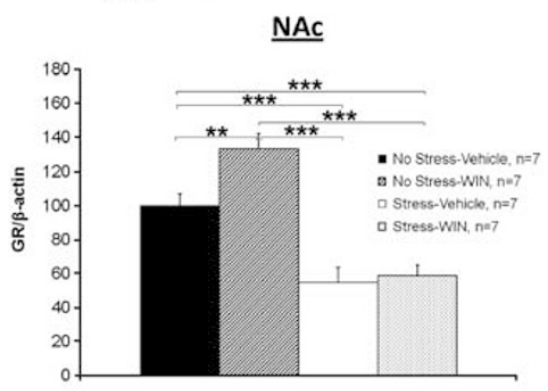

d

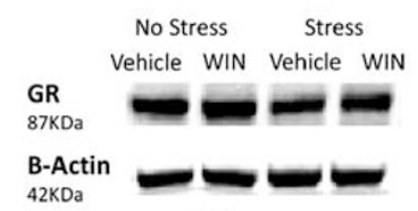

CA1

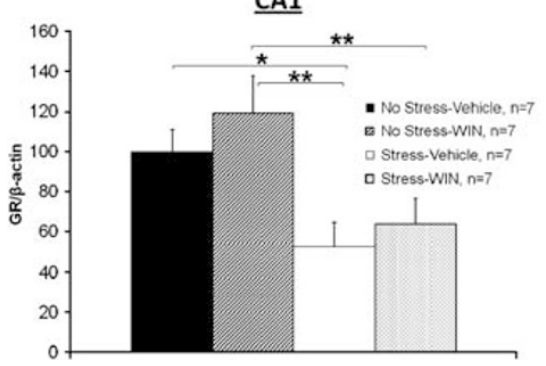

b
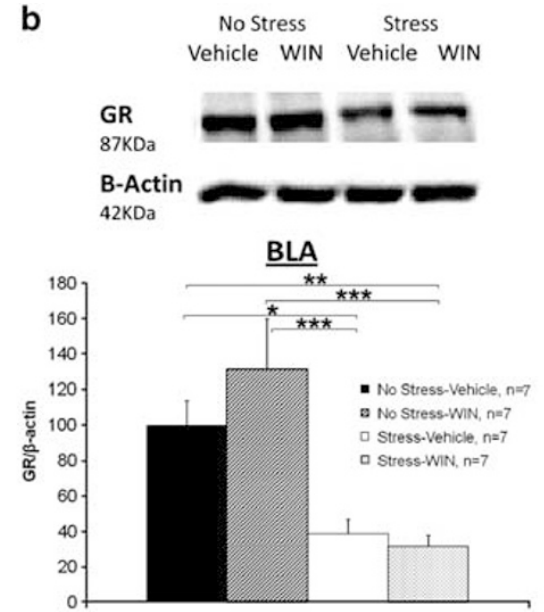

e
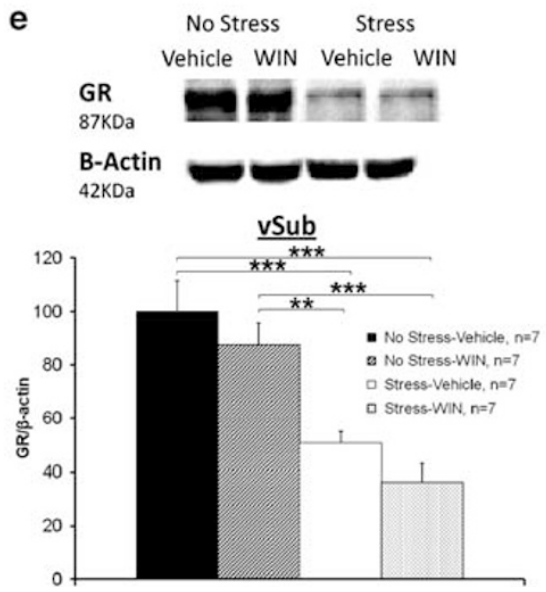

c
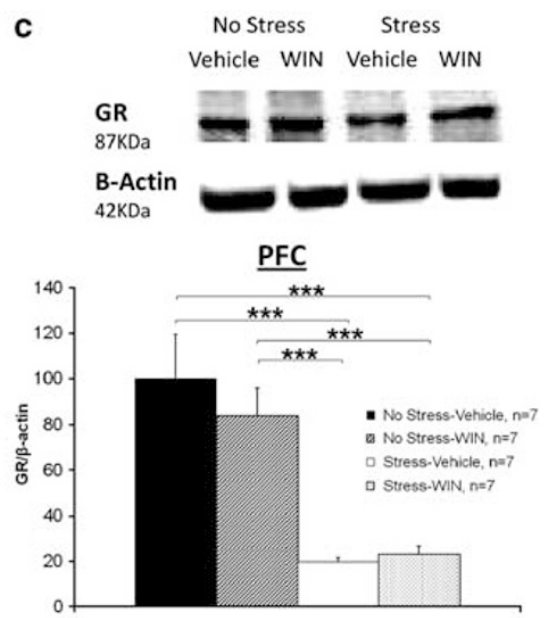

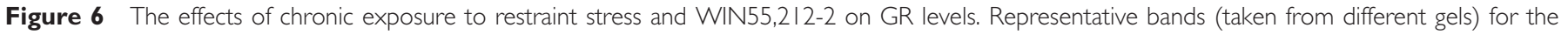

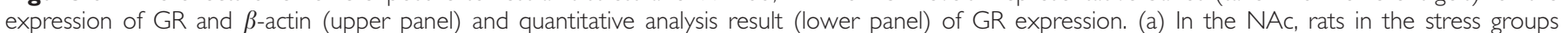

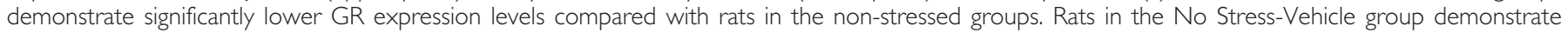

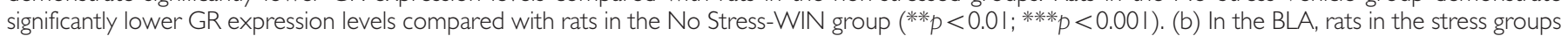

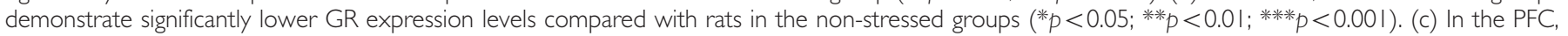

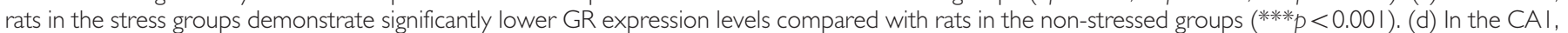

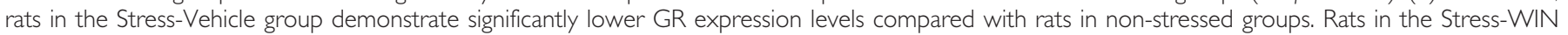

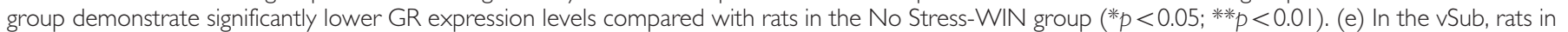
the stress groups demonstrate significantly lower GR expression levels compared with rats in the non-stressed groups (*** $<0.0$ I; **** $<0.00$ I).

Chronic WIN55,212-2 administration by itself also impaired performance, consistent with our previous findings of impaired short-term spatial location memory even after 75 days of withdrawal (Abush and Akirav, 2012). However, a combination of chronic WIN55,212-2 administration with chronic stress resulted in an intact spatial location memory.

It is interesting that WIN55,212-2 by itself impaired short-term location memory, but when administered in proximity to stress exposure, it attenuated the impairing effects of the stressor on memory. Hence, the effects of WIN55,212-2 on memory are dependent on the emotional state of the animal; this is supported by our previous findings that chronic WIN55,212-2 impaired short-term memory in the non-aversive spatial location task, but had no effect on spatial short-term memory in the aversive water-maze task (Abush and Akirav, 2010). Furthermore, we have shown that acute intra-BLAWIN55,212-2 can prevent the effects of acute elevated platform stress on performance in an aversive learning task (Ganon-Elazar and
Akirav, 2009), but acute WIN55,212-2 could not prevent the effects of the same stressor on the performance in a nonaversive task (Segev et al, 2012). In general, the cannabinoid system and the stress system are highly interconnected (Gorzalka et al, 2008; Hill and McEwen, 2010; Hill et al, 2010; Patel et al, 2004; Patel and Hillard, 2008), and it has been suggested that the endocannabinoid system might become activated specifically in highly aversive situations but not in non-aversive situations (Harloe et al, 2008; Holter et al, 2005; Niyuhire et al, 2007).

In the object-recognition task, stressed rats demonstrated attenuated exploration of the novel object compared with controls when tested 30 days after stress termination. This attenuated exploration was not observed when stress was accompanied with chronic WIN55,212-2 administration.

In the object-location task and LTP, rats that were exposed to WIN55,212-2 without stress exposure demonstrated intact learning (ie, were significantly different from the $50 \%$ chance level) and intact LTP (ie, were significantly 
different from the $100 \%$ baseline); however, they were not significantly different from any of the other groups. In the spatial task, on the other hand, rats that were exposed to WIN55,212-2 without stress exposure did not demonstrate intact learning (ie, were not significantly different from the $50 \%$ chance level). Hence, the long-term effects of chronic exposure to WIN55,212-2 depends on the specific task and the brain region that are being examined.

\section{GR Expression Levels in the Amygdala, Hippocampus, PFC, and NAc}

Chronic restraint stress reduced expression levels of GRs in the NAc, BLA, PFC, CA1, and vSub, consistent with animal models of chronic stress that demonstrated decreased hippocampal GRs (Sapolsky et al, 1984). The effect of chronic stress on expression levels of GRs is in line with the changes in circulating stress hormones in response to stress, as most likely stress hormone receptors will also gradually change in their expression and function (Sapolsky et al, 1984). In support, it has been shown that chronic treatment of rats with corticosterone or exposure to repeated restraint results in increased 2-AG content in the amygdala (Hill et al, 2005). This suggests that the effect of repeated restraint on amygdalar 2-AG content could be secondary to a persistent increase in glucocorticoid signaling (Patel and Hillard, 2008). Hence, high circulating levels of glucocorticoids can result in decreased numbers of available receptors that can significantly influence HPA axis activity. However, behavioral and hormonal responses to repeated and predictable exposure to homotypic stressors such as restraint exhibit habituation (ie, a progressive decrease in the expression of stress responses after repeated applications of the same stressor). Indeed, it has been demonstrated that repeated activation of the HPA axis by restraint stress resulted in a progressive decrease in plasma corticosterone levels with increasing numbers of restraint episodes (Patel et al, 2004).

Administration of chronic WIN55,212-2 together with stress exposure did not affect this stress-induced decline in GRs in all the brain areas examined, suggesting that the beneficial effects of WIN55,212-2 on memory and plasticity were not mediated by alterations in GR levels in the brain areas tested.

We also found that chronic administration of WIN55,212-2 alone resulted in higher expression levels of GRs in the NAc, $24 \mathrm{~h}, 10$ days, and 30 days after withdrawal, suggesting a stable and robust effect. Despite this increase in GR expression levels following chronic WIN55,212-2 administration, WIN55,212-2 did not normalize the stress-induced downregulation in GR levels.

Hence, the mechanism through which WIN55,212-2 prevents the stress-induced memory and plasticity impairments is yet to be determined. One possible explanation could be that WIN55,212-2 affects systems that are activated by stress stimulation before the activation of the HPA axis, such as CRH or norepinephrine.

\section{Anxiety, Locomotion, Hedonia, and Despair-Related Behavior}

Chronic exposure to restraint stress enhanced anxiety as tested in the open field 30 days after the stress ended.
However, chronic exposure to restraint stress had no effect on despair-like behavior as measured in the forced-swim test and the sucrose consumption test $24 \mathrm{~h}, 10$ days, or 30 days after the stress ended. In general, a number of studies revealed inconsistencies in the induction of hedonic deficit in chronic stress models in both rats and mice (for review, see Strekalova et al, 2011). Other chronic restraint studies showed that the intensity and duration of stress are critical in the development of depressive symptoms in rats as tested in the forced-swim test and sucrose tests (Suvrathan et al, 2010). As a whole, chronically stressed rats exhibit changes in immobility and sucrose consumption during the period of stress exposure; however, in many cases (depending on stress intensity and duration), these effects disappear with time. For example, rats were restrained $(6 \mathrm{~h} /$ day/ 21 days) and tested in the next days for anxiety and depressive-like symptoms. Chronic stress decreased time in the center arena of the open field, whereas there was no effect on the immobility index and sucrose preference (Huynh et al, 2011).

In determining the neurobiological responses to repeated and chronic psychological stress (Feder et al, 2009; Herman et al, 2008; McEwen, 2007), there is an impressive capability of individuals to habituate to repeated stress (Grissom and Bhatnagar, 2009; Marti and Armario, 1998). Hence, there is habituation to the effects of chronic stress over time or possibly due to recovery of a short-term effect, which has dissipated by the time of examination. Many chronic stress paradigms produce changes that are dynamic and reversible (Conrad et al, 1999; Sousa et al, 2000; Vyas and Chattarji, 2004), suggesting that behavioral recovery is possible. Nevertheless, the exposure to restraint stress had significant effects on weight gain and cognitive measures that suggest that some of the effects do not go through a habituation process and probably do not recover.

Although stressed rats demonstrated depressive- and anxiety-like symptoms as indicated by their reduced gain weight and reduced time in the center of the open field, chronic WIN55,212-2 administration did not prevent these effects. Increased anxiety 1 month after stress exposure suggests that the preventing effects of WIN55,212-2 on memory and plasticity were not due to the erasure of the stressful experience. This is consistent with our previous results that acute WIN55,212-2 administration in proximity to exposure to intense acute stress prevented the stress-induced impairment in extinction but not the heightened unconditioned anxiety that was measured in the open field and the light-dark tests (Ganon-Elazar and Akirav, 2012).

\section{Summary}

Deficits in learning and memory associated with frontal lobe and hippocampal dysfunction have been reported in affective disorders, which is often precipitated by stressful life events (Austin et al, 2001; Dolan, 2002; Drevets et al, 1997). We suggest that cannabinoids have beneficial effects in preventing at least some of the effects of chronic stress on cognitive processes. Overall, our findings support the development of strategies that target the endocannabinoid system in the search for novel approaches to improved therapy. 


\section{DISCLOSURE}

The authors declare no conflict of interest.

\section{REFERENCES}

Abush H, Akirav I (2010). Cannabinoids modulate hippocampal memory and plasticity. Hippocampus 20: 1126-1138.

Abush H, Akirav I (2012). Short- and long-term cognitive effects of chronic cannabinoids administration in late-adolescence rats. PLoS One 7: e31731.

Ahima RS, Harlan RE (1990). Charting of type II glucocorticoid receptor-like immunoreactivity in the rat central nervous system. Neuroscience 39: 579-604.

Aisa B, Elizalde N, Tordera R, Lasheras B, Del Río J, Ramírez MJ (2009). Effects of neonatal stress on markers of synaptic plasticity in the hippocampus: Implications for spatial memory. Hippocampus 19: 1222-1231.

Austin MP, Mitchell P, Goodwin GM (2001). Cognitive deficits in depression: possible implications for functional neuropathology. Br J Psychiatry 178: 200-206.

Belujon P, Grace AA (2008). Critical role of the prefrontal cortex in the regulation of hippocampus-accumbens information flow. Neurosci 28: 9797-9805.

Bortolato M, Mangieri RA, Fu J, Kim JH, Arguello O, Duranti A et al (2007). Antidepressant-like activity of the fatty acid amide hydrolase inhibitor URB597 in a rat model of chronic mild stress. Biol Psychiatry 62: 1103-1110.

Caspi A, Sugden K, Moffitt TE, Taylor A, Craig IW, Harrington H et al (2003). Influence of life stress on depression: moderation by a polymorphism in the 5-HTT gene. Science 301: 386-389.

Conrad CD, Galea LA, Kuroda Y, McEwen BS (1996). Chronic stress impairs rat spatial memory on the $\mathrm{Y}$ maze, and this effect is blocked by tianeptine pretreatment. Behav Neurosci 110: $1321-1334$.

Conrad CD, LeDoux JE, Magariños AM, McEwen BS (1999). Repeated restraint stress facilitates fear conditioning independently of causing hippocampal CA3 dendritic atrophy. Behav Neurosci 113: 902-913.

Diamond DM, Campbell AM, Park CR, Halonen J, Zoladz PR (2007). The temporal dynamics model of emotional memory processing: a synthesis on the neurobiological basis of stressinduced amnesia, flashbulb and traumatic memories, and the Yerkes-Dodson law. Neural Plast 2007: 60803.

Diamond DM, Park CR, Campbell AM, Woodson JC (2005). Competitive interactions between endogenous LTD and LTP in the hippocampus underlie the storage of emotional memories and stress-induced amnesia. Hippocampus 15: 1006-1025.

Dinan TG (2005). Stress: the shared common component in major mental illnesses. Eur Psychiatry 20: S326-S328.

Dolan RJ (2002). Emotion, cognition, and behavior. Science 298: 1191-1194.

Dong Z, Cao J, Xu L (2007). Opiate withdrawal modifies synaptic plasticity in subicular-nucleus accumbens pathway in vivo. Neuroscience 144: 845-854.

Drevets WC, Price JL, Simpson JR, Todd RD, Reich T, Vannier M et al (1997). Subgenual prefrontal cortex abnormalities in mood disorders. Nature 386: 824-827.

Feder A, Nestler EJ, Charney DS (2009). Psychobiology and molecular genetics of resilience. Nat Rev Neurosci 10: 446-457.

Foy MR, Baudry M, Foy JG, Thompson RF (2008). 17beta-estradiol modifies stress-induced and age-related changes in hippocampal synaptic plasticity. Behav Neurosci 122: 301-309.

Ganon-Elazar E, Akirav I (2009). Cannabinoid receptor activation in the basolateral amygdala blocks the effects of stress on the conditioning and extinction of inhibitory avoidance. J Neurosci 29: 11078-11088.

Ganon-Elazar E, Akirav I (2012). Cannabinoids prevent the development of behavioral and endocrine alterations in a rat model of intense stress. Neuropsychopharmacology 37: $456-466$.

Ganon-Elazar E, Akirav I (2013). Cannabinoids and traumatic stress modulation of contextual fear extinction and GR expression in the amygdala-hippocampal-prefrontal circuit. Psychoneuroendocrinology (e-pub ahead of print).

Garcia R, Musleh W, Tocco G, Thompson RF, Baudry M (1997). Time-dependent blockade of STP and LTP in hippocampal slices following acute stress in mice. Neurosci Lett 233: 41-44.

Gorzalka BB, Hill MN, Hillard CJ (2008). Regulation of endocannabinoid signaling by stress: implications for stress-related affective disorders. Neurosci Biobehav Rev 32: 1152-1160.

Grissom N, Bhatnagar S (2009). Habituation to repeated stress: get used to it. Neurobiol Learn Mem 92: 215-224.

Hammen C (2005). Stress and depression. Annu Rev Clin Psychol 1: 293-319.

Hammen C, Brennan PA, Shih JH (2004). Family discord and stress predictors of depression and other disorders in adolescent children of depressed and nondepressed women. J Am Acad Child Adolesc Psychiatry 43: 994-1002.

Harloe JP, Thorpe AJ, Lichtman AH (2008). Differential endocannabinoid regulation of extinction in appetitive and aversive Barnes maze tasks. Learn Mem 15: 806-809.

Herkenham M, Lynn AB, Little MD, Johnson MR, Melvin LS, de Costa BR et al (1990). Cannabinoid receptor localization in brain. Proc Natl Acad Sci USA 87: 1932-1936.

Herman JP, Flak J, Jankord R (2008). Chronic stress plasticity in the hypothalamic paraventricular nucleus. Prog Brain Res 170: 353-364.

Herman JP, Ostrander MM, Mueller NK, Figueiredo H (2005). Limbic system mechanisms of stress regulation: hypothalamopituitary-adrenocortical axis. Prog Neuropsychopharmacol Biol Psychiatry 29: 1201-1213.

Hill MN, Ho WS, Meier SE, Gorzalka BB, Hillard CJ (2005). Chronic corticosterone treatment increases the endocannabinoid 2-arachidonylglycerol in the rat amygdala. Eur J Pharmacol 528: 99-102.

Hill MN, McEwen BS (2010). Involvement of the endocannabinoid system in the neurobehavioural effects of stress and glucocorticoids. Prog Neuropsychopharmacol Biol Psychiatry 34: 791-797.

Hill MN, McLaughlin RJ, Bingham B, Shrestha L, Lee TT, Gray JM et al (2010). Endogenous cannabinoid signaling is essential for stress adaptation. Proc Natl Acad Sci USA 107: 9406-9411.

Hill MN, Tasker JG (2012). Endocannabinoid signaling, glucocorticoid-mediated negative feedback, and regulation of the hypothalamic-pituitary-adrenal axis. Neuroscience 204: 5-16.

Holter SM, Kallnik M, Wurst W, Marsicano G, Lutz B, Wotjak CT (2005). Cannabinoid CB1 receptor is dispensable for memory extinction in an appetitively-motivated learning task. Eur $J$ Pharmacol 510: 69-74.

Huynh TN, Krigbaum AM, Hanna JJ, Conrad CD (2011). Sex differences and phase of light cycle modify chronic stress effects on anxiety and depressive-like behavior. Behav Brain Res 222: 212-222.

Irwin MR, Miller AH (2007). Depressive disorders and immunity: 20 years of progress and discovery. Brain Behav Immun 21: 374-383.

Katona I, Rancz EA, Acsady L, Ledent C, Mackie K, Hajos N et al (2001). Distribution of CB1 cannabinoid receptors in the amygdala and their role in the control of GABAergic transmission. J Neurosci 21: 9506-9518. 
Keller MC, Neale MC, Kendler KS (2007). Association of different adverse life events with distinct patterns of depressive symptoms. Am J Psychiatry 164: 1521-1529.

Kim JJ, Diamond DM (2002). The stressed hippocampus, synaptic plasticity and lost memories. Nat Rev Neurosci 3: 453-462.

Kim KS, Han PL (2006). Optimization of chronic stress paradigms using anxiety- and depression-like behavioral parameters. J Neurosci Res 83: 497-507.

Lupien SJ, Maheu F, Tu M, Fiocco A, Schramek TE (2007). The effects of stress and stress hormones on human cognition: implications for the field of brain and cognition. Brain Cogn 65: 209-237.

Léna I, Dh tel H, Garbay C, Daugé V (2001). Involvement of D2 dopamine receptors in the opposing effects of two CCK-B agonists in a spatial recognition memory task: role of the anterior nucleus accumbens. Psychopharmacology (Berl) 153: 170-179.

Macri S, Laviola G (2004). Single episode of maternal deprivation and adult depressive profile in mice: interaction with cannabinoid exposure during adolescence. Behav Brain Res 154: 231-238.

Maroun M, Richter-Levin G (2003). Exposure to acute stress blocks the induction of long-term potentiation of the amygdalaprefrontal cortex pathway in vivo. J neurosci 23: 4406.

Marti O, Armario A (1998). Anterior pituitary response to stress: time-related changes and adaptation. Int J Dev Neurosci 16: 241-260.

Mathews A, MacLeod C (2005). Cognitive vulnerability to emotional disorders. Annu Rev Clin Psychol 1: 167-195.

McEwen BS (2007). Physiology and neurobiology of stress and adaptation: central role of the brain. Physiol Rev 87: 873-904.

Mitra R, Jadhav S, McEwen BS, Vyas A, Chattarji S (2005). Stress duration modulates the spatiotemporal patterns of spine formation in the basolateral amygdala. Proc Natl Acad Sci USA 102: 9371-9376.

Mizoguchi K, Yuzurihara M, Ishige A, Sasaki H, Chui DH, Tabira T (2000). Chronic stress induces impairment of spatial working memory because of prefrontal dopaminergic dysfunction. J Neurosci 20: 1568-1574.

Nestler EJ, Barrot M, DiLeone RJ, Eisch AJ, Gold SJ, Monteggia LM (2002). Neurobiology of depression. Neuron 34: 13-25.

Niyuhire F, Varvel SA, Thorpe AJ, Stokes RJ, Wiley JL, Lichtman AH (2007). The disruptive effects of the CB1 receptor antagonist rimonabant on extinction learning in mice are taskspecific. Psychopharmacology (Berl) 191: 223-231.

O'Donnell P, Grace AA (1995). Synaptic interactions among excitatory afferents to nucleus accumbens neurons: hippocampal gating of prefrontal cortical input. J Neurosci 15: 3622-3639.

Park CR, Campbell AM, Diamond DM (2001). Chronic psychosocial stress impairs learning and memory and increases sensitivity to yohimbine in adult rats. Biol Psychiatry 50: 994-1004.

Patel S, Hillard CJ (2008). Adaptations in endocannabinoid signaling in response to repeated homotypic stress: a novel mechanism for stress habituation. Eur J Neurosci 27: 2821-2829.

Patel S, Roelke CT, Rademacher DJ, Cullinan WE, Hillard CJ (2004). Endocannabinoid signaling negatively modulates stressinduced activation of the hypothalamic-pituitary-adrenal axis. Endocrinology 145: 5431-5438.

Patel S, Roelke CT, Rademacher DJ, Hillard CJ (2005). Inhibition of restraint stress-induced neural and behavioural activation by endogenous cannabinoid signaling. Eur J Neurosci 21: 1057-1069.

Poucet B, Lenck-Santini PP, Hok V, Save E, Banquet JP, Gaussier P et al (2004). Spatial navigation and hippocampal place cell firing: the problem of goal encoding. Rev Neurosci 15: 89-107.

Radecki DT, Brown LM, Martinez J, Teyler TJ (2005). BDNF protects against stress-induced impairments in spatial learning and memory and LTP. Hippocampus 15: 246-253.
Rademacher DJ, Meier SE, Shi L, Vanessa Ho WS, Jarrahian A, Hillard CJ (2008). Effects of acute and repeated restraint stress on endocannabinoid content in the amygdala, ventral striatum, and medial prefrontal cortex in mice. Neuropharmacology 54: 108-116.

Ramot A, Akirav I (2012). Cannabinoid receptors activation and glucocorticoid receptors deactivation in the amygdala prevent the stress-induced enhancement of a negative learning experience. Neurobiol Learn Mem 97: 393-401.

Robertson Blackmore EK, Stansfeld SA, Weller I, Munce S, Zagorski BM, Stewart DE (2007). Major depressive episodes and work stress: Results from a national population survey. Am J Public Health 97: 2088-2093.

Rothman SM, Mattson MP (2010). Adverse stress, hippocampal networks, and Alzheimer's disease. Neuromolecular Med 12: 56-70.

Sapolsky RM, Krey LC, McEwen BS (1984). Glucocorticoid-sensitive hippocampal neurons are involved in terminating the adrenocortical stress response. Proc Natl Acad Sci USA 81: 6174-6177.

Sargolini F, Roullet P, Oliverio A, Mele A (2003). Effects of intra-accumbens focal administrations of glutamate antagonists on object recognition memory in mice. Behav Brain Res 138: 153-163.

Schneider M, Koch M (2007). The effect of chronic peripubertal cannabinoid treatment on deficient object recognition memory in rats after neonatal mPFC lesion. Eur Neuropsychopharmacol 17: $180-186$

Schneider M, Schomig E, Leweke FM (2008). Acute and chronic cannabinoid treatment differentially affects recognition memory and social behavior in pubertal and adult rats. Addict Biol 13: 345-357.

Schneiderman N, Ironson G, Siegel SD (2005). Stress and health: psychological, behavioral, and biological determinants. Annu Rev Clin Psychol 1: 607-628.

Segev A, Ramot A, Akirav I (2012). Stress hormones receptors in the amygdala mediate the effects of stress on the consolidation, but not the retrieval, of a non aversive spatial task. PLoS One 7: e29988.

Sousa N, Lukoyanov NV, Madeira MD, Almeida OF, Paula-Barbosa MM (2000). Reorganization of the morphology of hippocampal neurites and synapses after stress-induced damage correlates with behavioral improvement. Neuroscience 97: 253-266.

Southwick SM, Vythilingam M, Charney DS (2005). The psychobiology of depression and resilience to stress: implications for prevention and treatment. Annu Rev Clin Psychol 1: 255-291.

Strekalova T, Couch Y, Kholod N, Boyks M, Malin D, Leprince P et al (2011). Update in the methodology of the chronic stress paradigm: internal control matters. Behav Brain Funct 7: 9.

Suvrathan A, Tomar A, Chattarji S (2010). Effects of chronic and acute stress on rat behaviour in the forced-swim test. Stress 13: 533-540.

van Eekelen JA, Kiss JZ, Westphal HM, de Kloet ER (1987). Immunocytochemical study on the intracellular localization of the type 2 glucocorticoid receptor in the rat brain. Brain Res 436: $120-128$.

Vyas A, Chattarji S (2004). Modulation of different states of anxiety-like behavior by chronic stress. Behav Neurosci 118: 1450-1454.

Willner P, Muscat R, Papp M (1992). Chronic mild stress-induced anhedonia: a realistic animal model of depression. Neurosci Biobehav Rev 16: 525-534.

Wilmouth CE, Spear LP (2009). Hedonic sensitivity in adolescent and adult rats: taste reactivity and voluntary sucrose consumption. Pharmacol Biochem Behav 92: 566-573.

Wood GE, Young LT, Reagan LP, Chen B, McEwen BS (2004). Stress-induced structural remodeling in hippocampus: 
prevention by lithium treatment. Proc Natl Acad Sci USA 101: 3973.

Wright RL, Conrad CD (2008). Enriched environment prevents chronic stress-induced spatial learning and memory deficits. Behav Brain Res 187: 41-47.

Yehuda R, Vermetten E, McFarlane A (2012). Understanding depression as it occurs in the context of post-traumatic stress disorder. Depress Res Treat 2012: 178261.
Zangen A, Nakash R, Overstreet DH, Yadid G (2001). Association between depressive behavior and absence of serotonin-dopamine interaction in the nucleus accumbens. Psychopharmacologia 155: 434-439.

Zoppi S, Pérez Nievas BG, Madrigal JL, Manzanares J, Leza JC, García-Bueno B (2011). Regulatory role of cannabinoid receptor 1 in stress-induced excitotoxicity and neuroinflammation. Neuropsychopharmacology 36: 805-818. 